\title{
It's a matter of style
}

\author{
Jonathan M. Chen, MD
}

\begin{abstract}
From the Congenital Cardiac Surgery, Seattle Children's Hospital, Seattle, Wash.
Disclosures: Author has nothing to disclose with regard to commercial support.

Received for publication Jan 27, 2017; accepted for publication Feb 6, 2017; available ahead of print March 2, 2017.

Address for reprints: Jonathan M. Chen, MD, Congenital Cardiac Surgery, Seattle Children's Hospital, 4800 Sand Point Way NE - RC2.820, Seattle, WA 98105 (E-mail: jmchen@uw.edu).

J Thorac Cardiovasc Surg 2017;153:1527

$0022-5223 / \$ 36.00$

Copyright (c) 2017 by The American Association for Thoracic Surgery

http://dx.doi.org/10.1016/j.jtcvs.2017.02.003
\end{abstract}

\section{"Fashion changes, but style endures"}

$$
\text { - Coco Chanel }
$$

Even to the troglodyte cardiac surgeon, it would seem selfevident that initiatives targeting "early" extubation in the neonate would reap tremendous benefits in the reduction of postoperative morbidity and overall lengths of stay. Indeed, it is increasingly in vogue to embrace immediate extubation in the operating room, or on arrival in the intensive care unit, despite patient factors previously considered contraindications (eg, newborns, long bypass times, circulatory arrest). ${ }^{1}$ In this issue of the Journal, investigators from the Pediatric Cardiac Critical Care Consortium (PC4) examined the flip side of this phenomenon: the incidence of extubation failure and its impact on outcomes. ${ }^{2}$ A total of 899 neonates were demonstrated to have an $11 \%$ rate of extubation failure, with a wide variation in range (5\%-22\%) among 14 PC4 centers. Only congenital airway anomaly was independently associated with extubation failure.

As with any large database analysis, the study is limited in its granularity: the definition of diaphragm paralysis and vocal cord dysfunction is only as good as the data input, and STAT categories were grouped for analysis. No variable was used to assess residual lesions, or renal insufficiency, and no standardized protocols were followed to help reduce practice variation. Most important, those patients extubated in the operating room were excluded, potentially biasing the cohort of eligible patients for analysis.

Although Bennyworth and colleagues ${ }^{2}$ sought to study a phenomenon, in fact what their investigation more accurately depicts is a decision (to reintubate). The variability in practice (demonstrated to be fourfold), or "high $\mathrm{O} / \mathrm{E}$ ratio" cannot be assumed to be an inadequacy of a specific center, as it may in fact reflect a more aggressive initial extubation policy, or perhaps a choice motivated by other factors not examined in this database. In this sense, the pejorative term "failure" perhaps itself assigns a performance value to what may be a subjective decision of the bedside provider; it is, in fact, a matter of style. Like other technical performance metrics recently proposed for reintubates? ${ }^{3}$

\section{References}

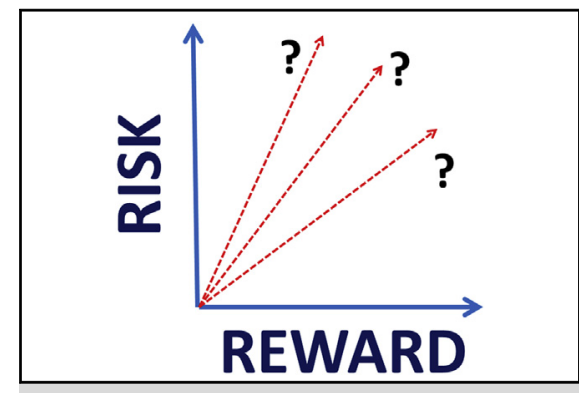

The choice of timing of extubation remains subjective as to risk/reward.

\section{Central Message}

The decision to extubate is one of risk/reward. Extubation "failure" is likely a marker of disease, and may or may not be causative of worse outcomes.

See Article page 1519.

congenital heart surgery, the extrapolation of "quality" based on an endpoint (reintubation) that itself is rooted in subjective assessment is thorny: is a center an "underperformer" if it aggressively attempts extubation in a marginal patient, understanding that there is a risk of reintubation? Conversely, is a center that nurses a patient along on nasal intermittent mandatory ventilation, or accepts a different level of ongoing tachypnea, "better" than one that simply

The decision to extubate is one of risk/reward, not unlike so many others in our field. This study suggests that congenital airway anomaly is worthy of consideration as it impacts that ratio, but to suggest that there is an "acceptable rate" of reintubation given the lack of specificity of the data would be a mistake. Extubation "failure" no doubt is a marker of disease, and may or may not be causative of worse outcomes. Fashion comes and fashion goes. What we need to take care to distinguish is whether these are truly best practices, or simply different ones.

1. Varghese J, Kutty S, Abdullah I, Hall S, Shostrom V, Hammel JM. Preoperative and intraoperative predictive factors of immediate extubation after neonatal cardiac surgery. Ann Thorac Surg. 2016;102:1588-95.

2. Bennyworth BD, Mastropietro CW, Graham EM, Klugman D, Costello JM Zhang W, et al. Variation in extubation failure rates after neonatal congenital heart surgery across Pediatric Critical Care Consortium (PC4) Hospitals. J Thorac Cardiovasc Surg. 2017;153:1519-26.

3. Bacha EA, Larrazabal LA, Pigula FA, Gauvreau K, Jenkins KJ, Colan SD, et al Measurement of technical performance in surgery for congenital heart disease: the stage I Norwood procedure. J Thorac Cardiovasc Surg. 2008;136:993-7. 